\title{
Pooled saliva samples as an approach to reduce the spread of infections with SARS-CoV-2
}

\author{
Johannes Möst ${ }^{1}\left[\right.$ D Angelika Eigentler $^{1} \cdot$ Dorothea Orth-Höller $^{1}$
}

Received: 10 February 2021 / Accepted: 20 February 2021 / Published online: 6 March 2021

(c) Springer-Verlag GmbH, DE part of Springer Nature 2021

To the editor,

For the detection of SARS-CoV-2 by PCR, saliva samples are at least as sensitive as nasopharyngeal samples [1]. Saliva samples have also been shown to be suitable for the screening of asymptomatic persons [2], and have been proposed as the key element for SARS-CoV-2 mass screening [3].

Saliva as a sample offers several advantages: it is easy to obtain, greatly reduces the nosocomial transmission risk, is better tolerated than the collection of a naso- or oropharyngeal swab leading to a good compliance, and poses no risk for complications.

Recently, Fogarty et al. suggested to use pooled saliva samples for surveillance testing health-care staff at least once a week [4]. Zhang et al. (medRxiv preprint https://doi. org/10.1101/2020.11.27.20240044) calculated that a routine testing frequency of at least once every 6 days, 4 days, and 2 days would be required to exceed pre-symptomatic case detection percentages of $33 \%, 50 \%$, and $67 \%$, respectively. Thus, to avoid introduction into an institution like a ward or a care home, testing should be done as frequently as possible.

Here, we report our experience with testing pooled saliva samples by PCR on a daily basis.

At the end of October 2020, we started testing all employees in our lab (28 persons). In the middle of December, we extended the testing to the staff of two care homes (71 and 45 persons, respectively).

Every morning, saliva samples are collected using a special device (Salivette ${ }^{\circledR}$, Sarstedt, Germany) [5]. With this system liquid, non-viscous saliva samples are obtained, which can be easily processed by an automated pipetting

Johannes Möst

jm@mb-lab.com

1 Clinical Microbiology Laboratory, MB-LAB,

Franz-Fischer-Straße 7b, 6020 Innsbruck, Austria system. Between 5 and 10 samples are pooled and tested by RT-PCR. Positive pools are splitted and reanalyzed immediately to identify the infected person(s).

Up to now, more than 400 pools with almost 2500 individual samples have been analyzed.

Nine of the pools (about 2.3\%) revealed invalid results. Since participants were tested daily, we decided to not retest these pools. Those persons who did not have a negative test result from the previous day underwent an antigen test from a nasopharyngeal swab.

So far, we have obtained two positive results-one in a technician in our lab and a second one in a nurse working in one of the care homes. Only two to three days later, both developed symptoms - the technician very mild, the nurse rather severe (high fever, cough, fatigue). Both would have worked for at least two more days and probably had infected others in the lab and the care home, respectively. No secondary infections and no other cases have occurred in all three institutions.

Our experience demonstrates that an approach with daily PCR testing of pooled saliva samples is feasible, well accepted by staff due to the comfortable sampling, and can contribute to the prevention of outbreaks in a vulnerable environment.

\section{Declarations}

Conflict of interest We declare that there are no conflicts of interests. No funding was received.

\section{References}

1. Medeiros da Silva RC, Nogueira Marinho LC, de Araújo Silva DN, Costa de Lima K, Pirih FQ, Luz de Aquino Martins AR. Saliva as a possible tool for the SARS-CoV-2 detection: a review. Travel Med Infect Dis. 2020;38:101920. https://doi.org/10.1016/j. tmaid.2020.101920. 
2. Yokota I, Shane PY, Okada K, Unoki Y, Yang Y, Inao T, et al. Mass screening of asymptomatic persons for SARS-CoV-2 using saliva. Clin Infect Dis. 2020. https://doi.org/10.1093/cid/ciaa1388.

3. Azzi L. Saliva is the key element for SARS-CoV-2 mass screening. Clin Infect Dis. 2020. https://doi.org/10.1093/cid/ciaa1440.

4. Fogarty A, Joseph A, Shaw D. Pooled saliva samples for COVID19 surveillance programme. Lancet Respir Med. 2020;8:1078-80. https://doi.org/10.1016/S2213-2600(20)30444-6.
5. Melo Villar L, Duarte da Costa V, Leires Marques BC, Lima da Silva L, Calheiros Santos A, da Fonseca Mendonça AC, et al. Usefulness of saliva samples for detecting SARS-CoV-2 RNA among liver disease patients. J Infect. 2021;82:e4-5. https://doi. org/10.1016/j.jinf.2020.07.017. 